Original Paper http://ajol.info/index.php/ijbcs http://indexmedicus.afro.who.int

\title{
Diversité floristique de la région de Foungbesso en zone de transition forêt - savane à l'ouest de la Côte d'Ivoire
}

\author{
Marie-Solange TIEBRE ${ }^{1 *}$, Djakalia OUATTARA ${ }^{1}$, Kouassi Bruno KPANGUI ${ }^{2}$, \\ Dido Fabrice KOUASSI ${ }^{1}$ et Kouakou Edouard N'GUESSAN ${ }^{1}$ \\ ${ }^{1}$ Laboratoire de Botanique, Université Félix Houphouët-Boigny, 22 BP 582 Abidjan 22, Côte d'Ivoire. \\ ${ }^{2}$ UFR Environnement, Université Jean Lorougnon Guédé, BP 150 Daloa, Côte d'Ivoire. \\ *Auteur correspondant ; E-mail : tiebre.ms@skynet.be ; Tél :0022546415181
}

\section{RÉSUMÉ}

L'analyse de la flore et de la végétation de la zone de transition forêt-savane, à l'ouest de la Côte d'Ivoire, dans la région de Foungbesso (départements de Touba et de Biankouma) a été réalisée. Des relevés de surfaces de $100 \mathrm{~m}^{2}$ et des relevés itinérants nous ont permis de recenser 8 différents biotopes, 349 espèces végétales reparties entre 259 genres et 76 familles botaniques. D’une façon générale, les milieux rencontrés sont floristiquement homogènes, en particulier les savanes herbeuses, les forêts claires, les forêts-galeries et les forêts denses semi-décidues. Cette étude a permis de dénombrer 09 espèces endémiques au bloc forestier ouest Africain (GCW), 03 espèces endémiques ivoiriennes (GCi) et 14 espèces identifiées comme rares et menacées selon l'UICN (2015). L'évaluation de la diversité floristique de la région de Foungbesso, à l'Ouest de la Côte d'Ivoire nous a permis de remarquer la présence d'une diversité de biotopes comme signalé dans la littérature mais en des superficies variables avec une prédominance des savanes.

(C) 2016 International Formulae Group. All rights reserved.

Mots clés : Diversité floristique, Ouest Côte d'Ivoire, végétation, zone de transition forêt - savane.

\section{Floristic diversity of Foungbesso region in the forest - savannah transition zone of western Côte d'Ivoire}

\section{ABSTRACT}

Analysis of the flora and vegetation of the transition zone forest-savannah in western Côte d'Ivoire in the region of Foungbesso was performed. Plots of vegetation of $100 \mathrm{~m}^{2}$ and itinerant relevé enabled to identify 8 different habitats, 349 plant species distributed among 259 genera and 76 botanical families. In general, the areas are floristically homogeneous, particularly grassland, woodland, gallery forest and semi-deciduous dense forests. This study enumerated 09 endemic species to the West African forest block (GCW), 03 Ivorian endemic species (CGi) and 14 species identified as rare and endangered by IUCN (2015). The evaluation of the floristic diversity of Foungbesso region allowed to note the presence of a diversity of habitats as reported in the literature but in varying size with a predominance of savannahs.

(C) 2016 International Formulae Group. All rights reserved.

Keywords: Floristic diversity, vegetation, Western Côte d'Ivoire, forest - savannah transition zone.

\section{INTRODUCTION}

Depuis son accession à l'indépendance en 1960, la Côte d'Ivoire a concentré son développement économique et social sur l'agriculture. Sa vaste couverture forestière, a fourni des terres arables qui ont favorisé la 
création de grandes plantations industrielles et villageoises de produits d'exportations (cacao, café, hévéa, palmiers à huile, etc.) mais aussi de cultures vivrières (banane, igname, manioc). Dans la partie ouest du pays, les forêts denses semi-décidues, les forêts claires et les savanes ont également fourni des devises à travers la culture du café, du cacaoyer et bien d'autres cultures. L'expansion de cette agriculture essentiellement de type itinérant sur brûlis a fortement contribué à la dégradation de la flore et de la végétation originelles, à une modification profonde du paysage et à la vulnérabilité de quelques espèces à statut particulier (Griffon, 2002 ; Teyssèdre, 2004). Il nous importe de faire un état des lieux de la flore et de la végétation de la Côte d'Ivoire (Koffi et al., 2015).

Les travaux de recherches floristiques dans la zone de transition forêt-savane à l'ouest de la Côte d'Ivoire se sont concentrés pour la plupart sur les aires protégées. Nous pouvons citer les travaux de Guillaumet et Adjanohoun (1971) sur la végétation de la Côte d'Ivoire, ceux de Lauginie (2007) sur la conservation de la nature et les aires protégées de Côte d'Ivoire, ceux de Tra Bi (1997) et Kouamé (1998) sur la flore de la forêt classée du haut-sassandra, ceux de Tra Bi (1997) et Nusbaumer et al. (2005) sur la forêt classée de Scio, et Goné Bi et al. (2013) sur le parc national du mont Péko. Dans les localités plus au nord, tel que Booro-Borotou et Touba, des travaux portant sur le bassin versant de cette région ont aussi été effectués par différents chercheurs (Chevallier, 1988 ; Boa, 1989 ; Camara, 1989 ; Planchon, 1989 ; Mitja, 1996 ; Akpesse et al., 2008). Des études additionnelles doivent être menées pour estimer la diversité floristique de la zone de transition forêt-savane de l'ouest de la Côte d'Ivoire, de faire un état des lieux des différents formations végétales rencontrées dans cette région et d'évaluer la valeur écologique de ces formations végétales pour le bien -être des populations locales (Koffi et al., 2015).

La présente étude se propose de contribuer à une meilleure connaissance de la flore et de la végétation de la zone de transition forêt-savane de l'ouest de la Côte d'Ivoire. De façon spécifique, il s'agit de quantifier la richesse et la composition spécifique et de caractériser la végétation de cette zone géographique en vue de montrer l'état de conservation de la flore et de la végétation dans cette zone de transition forêt savane.

\section{MATERIEL ET METHODES Site d'étude}

La localité de Foungbesso est située dans la partie ouest de la Côte d'Ivoire entre les latitudes Nord $7^{\circ} 58^{\prime} 30.0^{\prime \prime}$ et $7^{\circ} 53^{\prime} 42.0^{\prime \prime}$ et les longitudes Ouest $7^{\circ} 40^{\prime} 48.0^{\prime \prime}$ et $7^{\circ} 34^{\prime}$ 48.0". Elle est située à cheval entre les régions du Bafing (Département de Touba) et du Tonkpi (Département de Biankouma), plus précisément à $33 \mathrm{~km}$ de Biankouma et à environ $42 \mathrm{~km}$ de la ville de Touba (Figure 1). Selon Guillaumet et Adjanohoun (1971), Foungbesso est situé dans le secteur mésophile du domaine guinéen, une zone de transition entre la forêt dense humide sempervirente du domaine ombrophile et les forêts claires et les savanes du secteur subsoudanais. Le climax prédominant est la forêt dense humide semi-décidue. Les sols sont ferralitiques moyennement désaturés. Le réseau hydrographique est dominé principalement par la rivière Bafing et ses affluents. Les populations de la zone d'étude ont pour principale activité l'agriculture. Cette agriculture est fortement dominée par les cultures vivrières (le manioc, le taro, le riz et le maïs qui occupent une place de choix). Les cultures pérennes, quant à elles, sont constituées en grande partie de plantations de caféiers, de cacaoyers, d'anacardiers et d'hévéas.

\section{Collecte des données}

La collecte de données sur la flore a été réalisée sur la base d'une carte d'occupation du sol (Figure 1). Il s'agit de: forêt semidécidue, forêt claire, forêt galerie, savane boisée/arborée, savane arbustive, savane herbeuse, cultures et jachères. Pour l'inventaire floristique, nous avons combiné deux techniques de relevés à savoir, le relevé de surface et le relevé itinérant. 


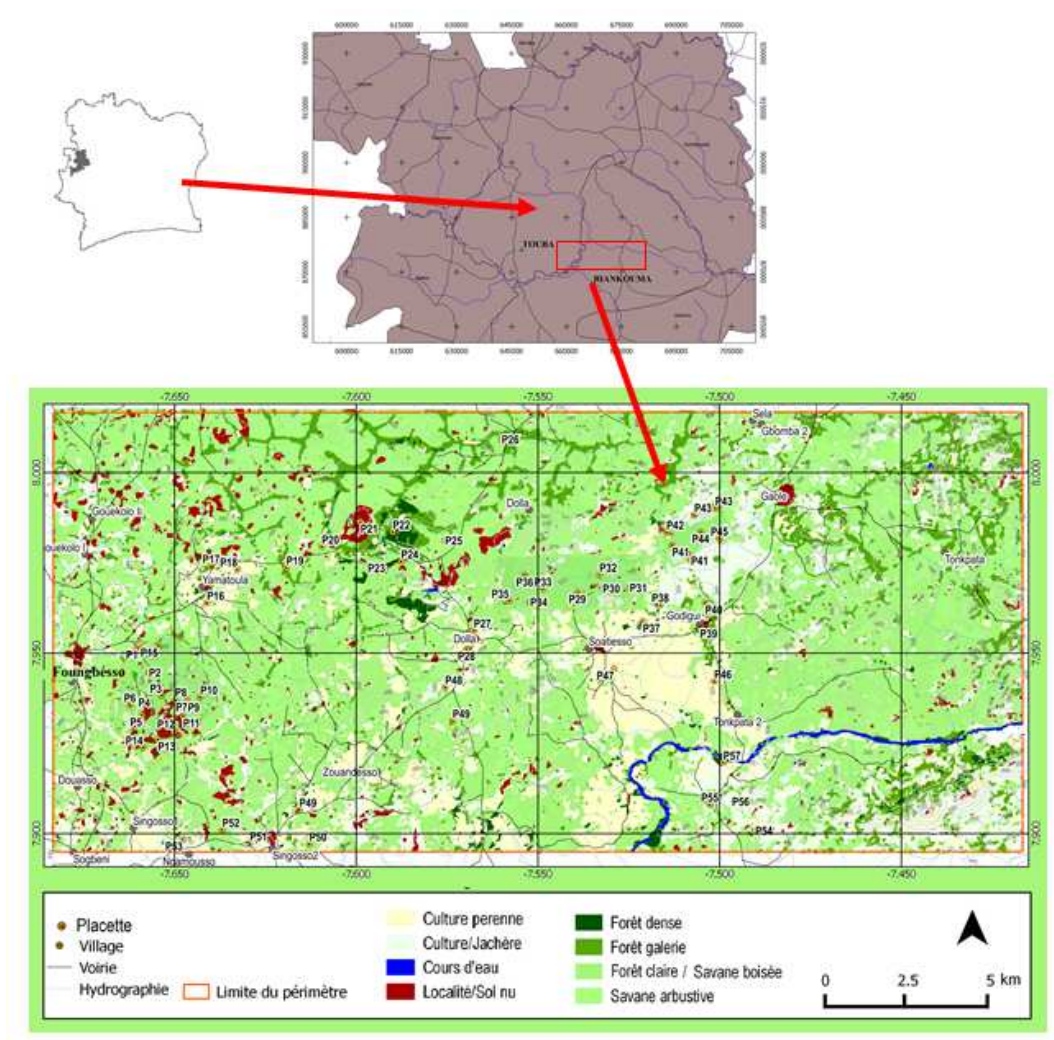

Figure 1 : Localisation de la zone d'étude et des relevés.

Le relevé de surface a consisté à délimiter des parcelles de $100 \mathrm{~m}^{2}(10 \mathrm{~m}$ x 10 m) dans chacun des types de biotope. A l'intérieur de chaque parcelle de $100 \mathrm{~m}^{2}$, toutes les espèces de plantes rencontrées ont été identifiées, leurs noms ont été notés et le nombre d'individus compté. Entre deux placettes, des inventaires itinérants ont été réalisés le long des pistes et des cours d'eaux, etc.

\section{Analyse des données \\ Diversité qualitative : Richesse floristique et composition floristique}

La richesse floristique est définie comme étant le nombre d'espèces recensées sur un territoire donné (Aké-Assi, 1984). Cette mesure permet d'établir la flore de la zone. La nomenclature selon Cronquist (1981) est celle qui a été suivie dans ce travail. L'analyse de la composition floristique a consisté à relever pour chaque espèce identifiée, le type biologique et l'affinité chorologique, qui renseigne sur l'origine des espèces. La liste générale des espèces recensées a été confrontée à celles de Aké Assi $(1984,2001,2002)$ pour identifier tous les paramètres de la composition floristique. Les espèces à statut particulier ont été mis en exergue en confrontant notre liste floristique à celle de Jongkind (2004) pour déterminer les espèces endémiques de Haute Guinée (HG), à ceux de Aké-Assi (1998) et de l'UICN (2015), pour la détermination des espèces rares et menacées d'extinction de la flore ivoirienne.

\section{Diversité quantitative: Indice de diversité} ( $H$ ') de Shannon

L'indice de diversité de Shannon mesure la diversité spécifique d'un peuplement, en combinant l'abondance relative des espèces et la richesse spécifique. Il a été utilisé pour évaluer puis comparer la richesse floristique des différents biotopes de notre zone d'étude. Si nous désignons par $N$ la fréquence totale des $S$ espèces considérées, $n i$ la fréquence d'une espèce $i$ et $P i(n i / N)$ la fréquence relative de l'espèce $i$, alors l'indice de Shannon se résume à l'expression mathématique suivante : $\mathrm{H}^{\prime}=-\sum$ [pi $\left.\mathrm{x} \ln \mathrm{pi}\right]$. Les valeurs de cet indice varient entre 0 et $\ln$ 
$\mathrm{N}$, avec ln $\mathrm{N}$ dépassant rarement 5 (Felfili et al., 2004).

\section{Indice d'équitabilité (E) de Piélou}

L'équitabilité $E$ s'obtient en rapportant la diversité observée à la diversité théorique maximale. L'équitabilité varie de 0 à 1 . Elle tend vers 0 quand la quasi-totalité des effectifs est concentrée sur une espèce et vers 1 lorsque toutes les espèces ont la même abondance. Dans le cas où cet indice tend vers 1 , le milieu en question est dit équilibré. L'indice d'équitabilité se calcule selon la formule mathématique suivante : $E=H^{\prime} / \ln S$ où $E$ désigne l'indice de Piélou (1966), H' désigne l'indice de Shannon, $S$ le nombre total d'espèces de la plantation autre que le cacaoyer, et $\operatorname{lnS}$ représente la diversité maximale du biotope.

\section{Analyse statistique des données}

L'analyse de variance (ANOVA) a été utilisée pour comparer les moyennes des indices calculées des différents biotopes étudiés. L'ANOVA compare la variance intragroupe à la variance intergroupe. Il s'agit d'une analyse paramétrique, c'est-à-dire que l'on considère que la variable mesurée a une distribution normale. Le but de l'analyse est d'établir si les moyennes des valeurs mesurées dans différents groupes, sont significativement différentes. Elle repose sur trois principes : l'indépendance des observations, la normalité de la distribution, et l'homogénéité des variances (l'homoscédasticité). La normalité est vérifiée par le test de Shapiro-Wilk. L'homogénéité des variances a été vérifiée par le test de Levene. Le niveau de significativité choisi pour ces analyses, est de 5 p.c. $(P=$ $0,05)$. Chaque fois que la probabilité calculée était significative, le test de Tukey est effectué pour comparer deux à deux les moyennes et apprécier les différences significatives qui existent entre celles-ci. Le logiciel XLSTAT 2014 a été utilisé pour la réalisation de ces tests statistiques.

\section{RESULTATS \\ Richesse et composition floristique}

Les travaux de terrain ont permis d'échantillonner au total 57 relevés de surface repartis à travers les différents biotopes. Concernant la richesse floristique, ce sont au total 349 espèces végétales reparties en 256 genres et 76 familles botaniques qui ont été recensées. De ces 256 genres, 56 comprennent au moins deux (2) espèces soit $22 \%$ et 200 soit $78 \%$ ne contiennent qu'une seule espèce. Les genres les plus représentés sont: Ficus $(9$ espèces), Vernonia (6 espèces), Combretum (5 espèces), Sida (5 espèces), et Cissus, Cola, Pennisetum, et Setaria (4 espèces chacune). Les familles les plus riches en espèces sont celles des Poaceae (29 espèces), des Fabaceae (27 espèces), des Rubiaceae (24 espèces) et des Euphorbiacae (18 espèces) avec respectivement $8 \%, 8 \%, 7 \%$ et $5 \%$.

Parlant des biotopes, les forêts galeries sont celles dans lesquelles l'on a rencontré le plus grand nombre d'espèces (123) tandis que les savanes herbeuses sont celles dans lesquelles le moins d'espèces (24) ont été rencontrées (Tableau 1). Les familles botaniques les mieux représentées diffèrent par biotope. La famille des Fabaceae est celle regroupant le plus d'espèces dans les savanes boisée/arborée, les forêts galeries et les cultures tandis que celle des Poaceae regroupe le plus d'espèces dans les savanes arbustives, les jachères et les savanes herbeuses. Quant aux forêts claires et aux forêts semi-décidues, les familles regroupant le plus d'espèces sont respectivement les Euphorbiaceae et les Sapindaceae (Figure 2).

\section{Diversité qualitative de la zone d'étude}

Pour l'ensemble de la zone d'étude, concernant l'affinité chorologique des espèces, ce sont les espèces GuinéoCongolaises Soudano-Zambéziene (GC-SZ) qui sont les plus représentées avec $46,6 \%$. Elles sont suivies des espèces de la région Guinéo-Congolaises (GC) avec 37,7\%. Les espèces endémiques à la Côte d'Ivoire (GCi) sont les moins représentées avec $0,9 \%$ de l'ensemble des espèces recensées. Les espèces du bloc GC sont les plus nombreuses dans les forêts semi-décidues et les cultures avec respectivement $66,7 \%$ et $53,1 \%$ tandis qu'elles le sont moins dans les autres biotopes en remplacement de celle du bloc GC-SZ. Les savanes herbeuses sont celles qui comportent le plus d'espèces du bloc GC-SZ avec une proportion de 70,8\% (Figure 3).

$\mathrm{Au}$ niveau du type biologique, ce sont les arbres mésophanérophytes qui sont les plus représentés dans l'ensemble du site d'étude avec une proportion de 43,9\%. Ils sont suivis par les lianes mésophanérophytes 
$(9,8 \%)$. A l'exception des savanes herbeuses dans lesquelles l'on ne retrouve aucun arbre mésophanérophyte, les autres biotopes en comportent en proportion plus élevée. Les cultures par exemple comportent $75 \%$ d'arbres mésophanérophytes. Les thérophytes ne sont présents que dans les savanes herbeuses, les cultures et les jachères avec une plus grande proportion dans les savanes herbeuses $(20,83 \%)$ et, à l'inverse une faible proportion dans les cultures $(6,25 \%)$. Les lianes microphanérophytes par contre ne sont présentes que dans les jachères avec une proportion de 17,14\% (Figure 4).

\section{Espèces à statut particulier de la zone d'étude}

Parmi les espèces recensées, neuf (9) espèces sont signalées comme endémiques au bloc forestier Ouest africain (GCW). Il s'agit de Eriosema molle, Leptoderris cyclocarpa, Mussaenda grandiflora, Napoleona ealeonensis, Premna hispida, Samanea dinklagei, Tetracera affinis, Tricalysia discolor, Tristemma coronatum selon les listes de Aké-Assi (2001; 2002). Nous avons aussi comptabilisé trois (3) espèces endémiques de Côte d'Ivoire (GCi). Ce sont: Baphia bancoensis, Hibiscus comoensis et Uvaria tortilis. L'étude a permis d'identifier 14 espèces considérées comme rares et menacées selon l'UICN (2015). Elles sont de la classe des espèces vulnérables pour 4 d'entre elles. Ce sont: Afzelia africana, Albizia ferruginea, Khaya grandifoliola, et Mitragyna ledermannii. Ces espèces appartiennent à la classe des taxons dont le risque d'extinction est toujours croissant. Quatre (4) espèces sont considérées comme menacées d'extinction selon les listes de Aké-assi (1998). Ce sont : Hibiscus comoensis, Lannea nigritana, Milicia excelsa et Uvaria tortilis (Tableau 2).

\section{Diversité quantitative de la zone d'étude}

Les indices de diversité de Shannon dans l'ensemble de la zone d'étude sont

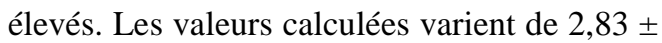
0,41 (savane herbeuse) à 4,71 $\pm 0,06$ (forêt galerie). Les valeurs de l'indice de Shannon des autres biotopes sont comprises entre ces deux valeurs. Les différences entre les valeurs moyennes sont significatives $(\mathrm{F}=76,03 ; p<$ 0,0001). Lorsqu'on considère l'indice d'équitabilité de Piélou, les valeurs sont également élevées dans l'ensemble de la zone d'étude. Elles varient de 0,69 $\pm 0,02$ (savane herbeuse) à $0,95 \pm 0,02$ (forêt semi-décidue). Il existe également une différence significative entre ces valeurs moyennes $(\mathrm{F}=$ 11,37; $p<0,0001$ ) (Tableau 3).
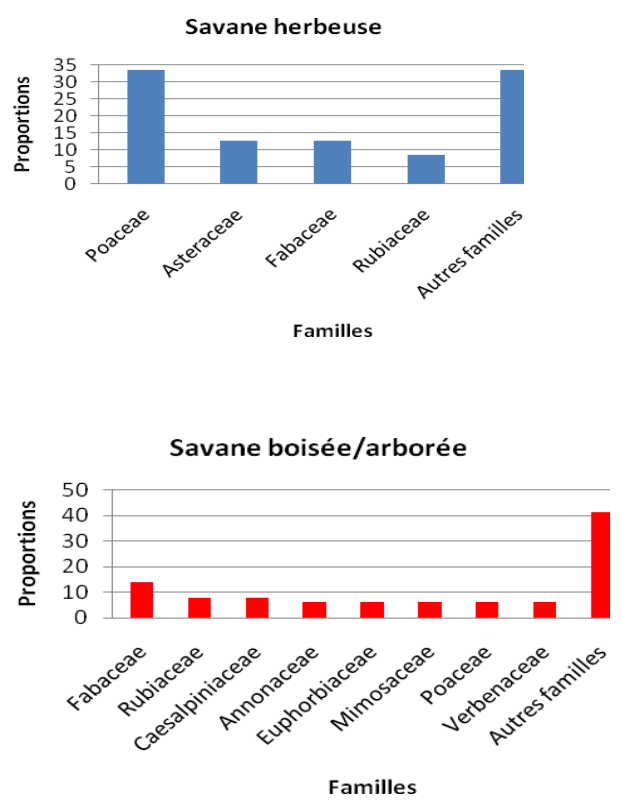
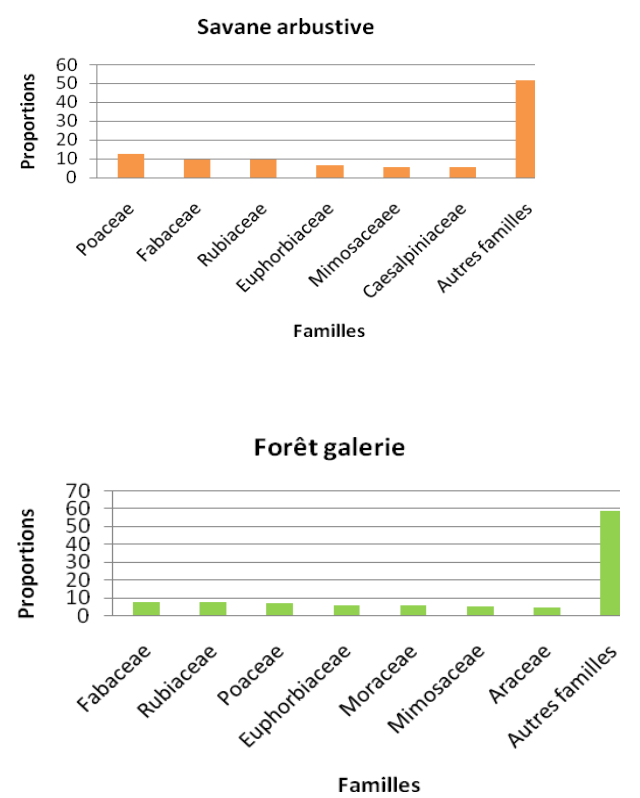


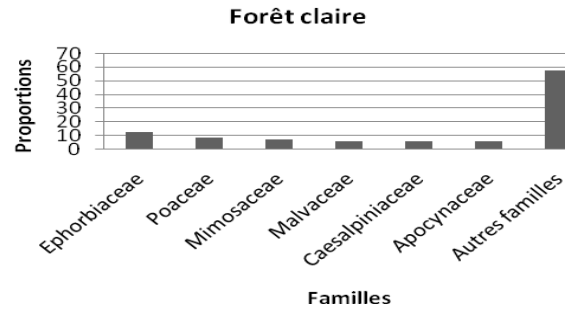

Cultures

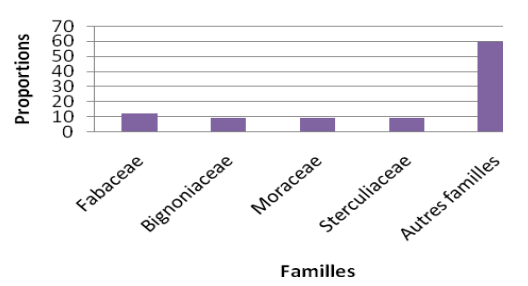

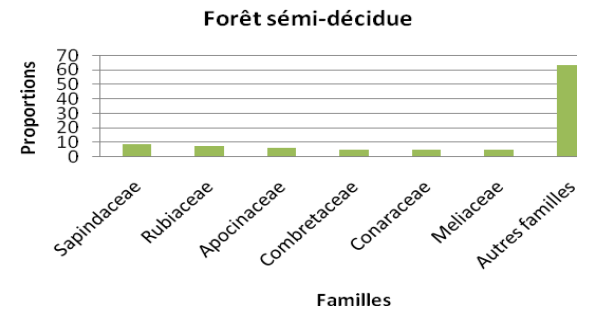

Jachère

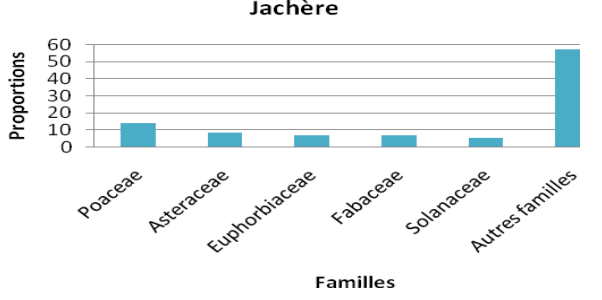

Figure 2 : Répartition des familles en fonction du biotope dans la zone d'étude.

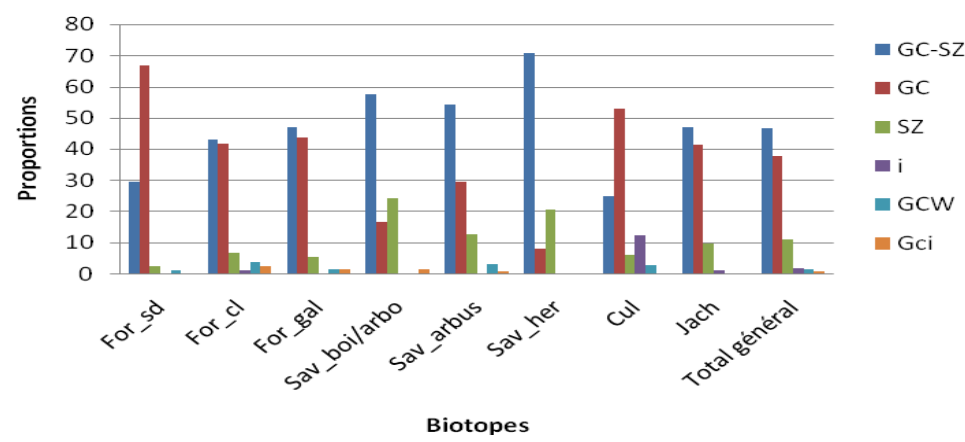

Figure 3 : Distribution des types chorologiques en fonction des biotopes dans la zone d'étude. For_sd : forêt semi-décidue ; For_cl : forêt claire ; For_gal : forêt galerie ; Sav_boi/arbo : savane boisée/arborée ; Sav_arbus : savane arbustive ; Sav_her : savane herbeuse ; Cul : cultures ; Jach : jachère.

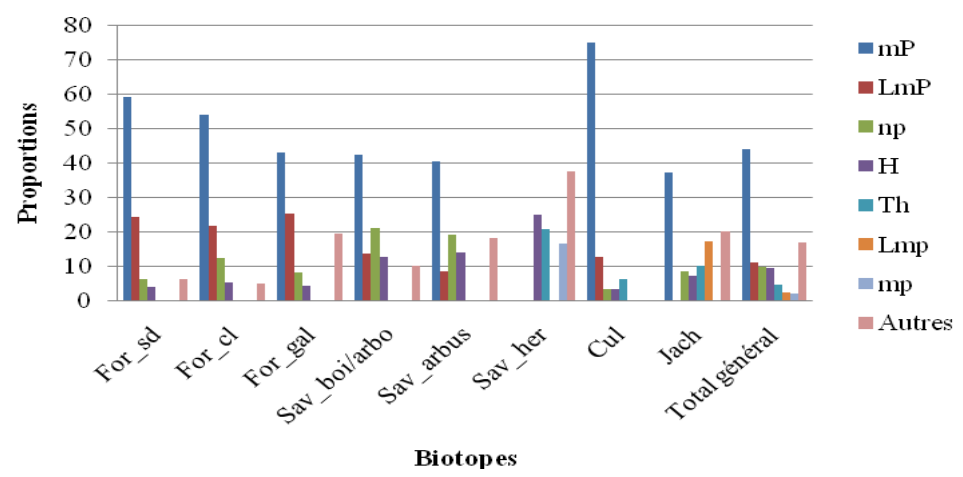

Figure 4 : Distribution des types biologiques en fonction des biotopes dans la zone d'étude. For_sd : forêt semi-décidue ; For_cl : forêt Claire ; For_gal : forêt galerie ; Sav_boi/arbo : savane boisée/arborée ; Sav_arbus : savane arbustive ; Sav_her : savane herbeuse ; Cul : cultures ; Jach : jachère. 
Tableau 1 : Richesse floristique par biotope dans la zone d'étude.

\begin{tabular}{lcccc}
\hline Biotopes & Nombre & Nombre & & Familles \\
\cline { 5 - 5 } & d'espèces & de genres & Nombre & Familles représentatives \\
\hline Forêt semi-décidue & 81 & 73 & 37 & Sapindaceae et Rubiaceae \\
Forêt claire & 75 & 68 & 34 & Euphorbiaceae et Poaceae \\
Forêt galerie & 123 & 103 & 54 & Fabaceae et Rubiaceae \\
Savane boisée/arborée & 66 & 61 & 31 & Fabaceae et Rubiaceae \\
Savane arbustive & 95 & 89 & 39 & Poaceae et Fabaceae \\
Savane herbeuse & 24 & 21 & 12 & Poaceae et Asteraceae \\
Cultures & 32 & 31 & 18 & Fabaceae et Bignoniaceae \\
Jachère & 70 & 59 & 36 & Poaceae et Asteraceae \\
Total & 349 & 256 & 76 & Poaceae et Fabaceae \\
\hline
\end{tabular}

Tableau 2 : Liste des espèces à statut particulier de la zone d'étude.

\begin{tabular}{lc}
\hline Espèces & Statut \\
\hline Afzelia africana & VU \\
Albizia ferruginea & VU \\
Argocoffeopsis afzelii & HG \\
Baphia bancoensis & GCi \\
Cissus doeringii & LC \\
Commelina erecta & LC \\
Desmodium salicifolium & LC \\
Dicliptera elliotii & LC \\
Eriosema molle & GCW \\
Haemanthus multiflorus & HG \\
Hibiscus comoensis & HG_GCi \\
Khaya grandifoliola & VU \\
Lannea nigritana & Rare \\
Leptoderris cyclocarpa & HG_GCW \\
Milicia excelsa & LR/LC_rare \\
Millettia zechiana & LC \\
Mitragyna ledermannii & VU \\
Mussaenda grandiflora & GCW \\
Panicum repens & LC \\
Premna hispida & GCW \\
Pterocarpus santalinoides & LR/LC \\
Samanea dinklagei & GCW \\
Tacca leontopetaloides & LC \\
Tetracera affinis & GCW \\
Tricalysia discolor & GCW \\
Triplochiton scleroxylon & LR/LC \\
Tristemma coronatum & GCW \\
Turraea heterophylla & HG \\
Uvaria tortilis & HG_Gci_rare \\
VU : vulnérable ; HG : endémique de Haute Guinée ; \\
LC : préoccupation mineure; LR : risque mineur ; \\
GCi ; endémique à la Côte d'Ivoire ; \\
GCW : endémique au Bloc forestier Ouest africain. \\
\\
\end{tabular}


Tableau 3 : Indices de diversité calculés pour les types de végétation rencontrés dans la zone d'étude.

\begin{tabular}{lccccccccc}
\hline Indices & Sav_her & $\begin{array}{c}\text { Sav_arb } \\
\text { us }\end{array}$ & Cul & Jach & $\begin{array}{c}\text { Sav_boi/ } \\
\text { arbo }\end{array}$ & For_cl & For_sd & For_gal & $\begin{array}{c}\text { Statistique } \\
\text { du test }\end{array}$ \\
\hline Diversité de & $2,83 \pm$ & $3,08 \pm$ & $3,12 \pm$ & $3,15 \pm$ & $3,25 \pm$ & $3,99 \pm$ & $4,38 \pm$ & $4,71 \pm$ & $\mathrm{F}=76,03$ \\
Shannon & $0,41^{\mathrm{a}}$ & $0,45^{\mathrm{a}}$ & $0,94^{\mathrm{a}}$ & $0,39^{\mathrm{a}}$ & $0,22^{\mathrm{ab}}$ & $0,24^{\mathrm{bc}}$ & $0,32^{\mathrm{c}}$ & $0,06^{\mathrm{c}}$ & $p<0,0001$ \\
\hline Equitabilité & $0,69 \pm$ & $0,79 \pm$ & $0,89 \pm$ & $0,92 \pm$ & $0,91 \pm$ & $0,92 \pm$ & $0,95 \pm$ & $0,88 \pm$ & $\mathrm{F}=11,37$ \\
de Piélou & $0,02^{\mathrm{a}}$ & $0,03^{\mathrm{b}}$ & $0,03^{\mathrm{c}}$ & $0,3^{\text {cd }}$ & $0,2^{\text {cd }}$ & $0,02^{\text {cd }}$ & $0,02^{\mathrm{d}}$ & $0,01^{\mathrm{c}}$ & $p<0,0001$ \\
\hline
\end{tabular}

Pour chaque ligne, les valeurs suivies par une même lettre ne sont pas significativement différentes au seuil de $5 \mathrm{p}$. c. For_sd : forêt semi-décidue ; For_cl : forêt Claire ; For_gal : forêt galerie ; Sav_boi/arbo : savane boisée/arborée ; Sav_arbus : savane arbustive ; Sav_her : savane herbeuse ; Cul : cultures ; Jach : jachère.

\section{DISCUSSION}

La présente étude a permis de mettre en évidence la plupart des formations végétales répertoriées dans la littérature (Guillaumet et Adjanohoun, 1971; Lauginie, 2007). Les types de formations végétales varient des forêts denses semi-décidues aux savanes herbeuses en passant par les jachères et les cultures. Il existe toujours une grande diversité écosystémique dans la zone d'étude, mais il faut noter que ces végétations existent en taille variable sur le terrain. Les savanes arbustives, les zones cultivées et les jachères sont de loin les plus représentatives de la zone d'étude. Elles représentent à elle seule 52\% des parcelles inventoriées au cours de cette étude, démontrant que l'activité humaine est remarquable dans la zone. La végétation est défrichée pour laisser place aux cultures vivrières et pérennes abondantes et variées (Lykke, 1998 ; Lykke et Sambou, 1998 ; Lykke et Sambou, 2002). Les forêts denses semi-décidues et les forêts-galeries sont peu abondantes dans la zone et pour leur grande majorité aussi défrichées pour laisser place aux cultures pérennes de caféiers et de cacaoyers. La végétation forestière du site est donc très fragmentée par les activités humaines.

Les 349 espèces recensées dans l'ensemble du site sont aussi révélatrices d'une diversité végétale peu élevée. En effet, lorsqu'on compare ces données avec la diversité floristique de la Côte d'Ivoire selon Aké-Assi (2001, 2002), l'on s'aperçoit que la contribution de la zone de Foungbesso à la flore générale ivoirienne est de 09,05\% au niveau des espèces, $20,15 \%$ au niveau des genres et de $38,97 \%$ au niveau des familles. Les familles botaniques dominantes sont les
Poaceae, les Fabaceae et les Rubiaceae. Cette situation est analogue à celles rapportées par d'autres chercheurs (Tra Bi, 1997; Kouamé, 1998; Nusbaumer et al., 2005; Koulibaly, 2008; Goné Bi et al., 2013) pour le secteur mésophile $\mathrm{du}$ domaine guinéen. $\mathrm{La}$ prédominance des mésophanérophytes $(43,9 \%)$ serait due à l'action des feux de brousse qui ravagent presque tous les ans la zone, ne laissant que les arbres au détriment des autres types biomorphologiques (Lykke, 1998 ; Lykke et Sambou, 1998 ; Lykke et Sambou, 2002 ; Sangne, 2009).

En ce qui concerne le cortège d'espèces à statut particulier, l'étude a permis d'en répertorier 29. Ces plantes endémiques ouestafricaines, ivoiriennes, rares et vulnérables sont reparties sur presque toute la zone. Goné $\mathrm{Bi}$ et al. (2013) ont identifiés 74 espèces endémiques, rares et menacées d'extinction dans le parc national du Mont Péko. La différence entre ces deux études peut s'expliquer par le fait que le parc national du Mont Péko, plus riche en espèces, est situé plus au sud du secteur mésophile comparé à la région de Foungbesso. Ces résultats corroborent ceux de Guillaumet et Adjanohoun (1971) qui mentionnent que le secteur mésophile est relativement moins diversifié en allant du sud plus forestier vers le nord savanicole. Les espèces endémiques, rares et menacées d'extinction sont celles pour lesquelles les efforts de conservation doivent être prioritaires (Myers et al., 2000). Rencontrer des espèces de ce genre dans le site d'étude montre sa diversité et l'attention que l'on devrait accorder aux formations végétales où elles sont présentes. 


\section{Conclusion}

L'évaluation de la diversité floristique de la région de Foungbesso en zone de transition forêt-savane à l'ouest de la Côte d'Ivoire a permis d'identifier la présence d'une diversité de biotopes comme signalé dans la littérature mais en des superficies variables avec une prédominance des savanes. Ce sont 349 espèces reparties en 256 genres et 76 familles botaniques qui ont pu être recensées. Parmi ces 349 espèces, 09 espèces sont endémiques au bloc forestier Ouest Africain, 03 espèces sont endémiques ivoiriennes et enfin 14 sont rares selon les listes de l'UICN. L'on retiendra que la végétation est très perturbée par les activités humaines.

\section{REMERCIEMENTS}

Nos remerciements vont à l'endroit de Monsieur Assi Yapo Jean du Centre National de Floristique de Côte d'Ivoire pour la détermination des échantillons d'herbiers. Les auteurs tiennent aussi à remercier les trois référés pour leurs remarques constructives qui ont permis l'amélioration du document.

\section{REFERENCES}

Aké-Assi L. 1984. Flore de la Côte d'Ivoire. Thèse de Doctorat, Univ. Abidjan.

Aké-Assi L. 1998. Impact de l'exploitation forestière et du développement agricole sur la conservation de la biodiversité biologique en Côte d'Ivoire. Le Flamboyant, 46 : 20-21.

Aké-Assi L. 2001. Flore de la Côte d'Ivoire : catalogue systématique, biogéographie et écologie. Mémoire de Botanique systématique. Conservatoire et Jardin Botanique de Genève ; Boisseria 57.

Aké-Assi L. 2002. Flore de la Côte d'Ivoire : catalogue systématique, biogéographie et écologie. Mémoire de Botanique systématique. Conservatoire et Jardin Botanique de Genève ; Boisseria 58.

Akpesse AA, Kouassi PK, Tano Y, Lepage M. 2008. Impact des termites dans les champs paysans de riz et de mais en savane sub-soudanienne (BooroBorotou, Côte-d'Ivoire) Sciences \& Nature, 5(2) : 121 - 131.

Boa D. 1989. Caractérisations, propriétés hydrodynamiques, contraintes et potentialités des sols gravillonnaires : cas de Booro-Borotou (région de Touba, nord-ouest la Côte-d'Ivoire). Thèse de $3 \mathrm{e}$ cycle, Université d'Abidjan.

Camara M. 1989. Les systèmes de culture et leurs influences sur quelques propriétés physiques et hydrodynamiques du sol : cas de Booro-Borotou (région de Touba, nord-ouest la Côte-d'Ivoire). Thèse de $3 \mathrm{e}$ cycle, Université d'Abidjan.

Chevallier P. 1988. Complexité hydrologique du petit bassin versant. Exemple en savane humide Booro-Borotou (Côte d'Ivoire). Thèse de doctorat, U.S.T.L., Montpellier.

Cronquist A. 1981. An Integrated System of Classification of Flowering Plants. Columbia University Press.

Felfili JM, Silva Júnior MC, Sevilha AC, Fagg CW, Walter BMT, Nogueira PE, Rezende AV. 2004. Diversity, floristic and structural patterns of cerrado vegetation in Central Brazil. Plant Ecology, 175: 37-46.

Goné BZB, Koamé D, Koné I, Adou Yao CY. 2013. Diversité végétale et valur de conservation pour la biodiversité du Parc National du Mont Péko, une aire protégée, menacée de disparition en Côte d'Ivoire. Journal of Applied Biosciences., 71: 5753-5762.

Griffon M. 2002. Révolution Verte, Révolution Doublement Verte Quelles technologies, institutions et recherche pour les agricultures de l'avenir? Mondes en Développement, 1: 39-44.

Guillaumet JL, Adjanohoun E. 1971. La végétation. In Avenard JM (ed) Le milieu naturel de la Côte d'Ivoire. Mémoires ORSTOM, 50: 157-262.

Hiernaux P. 1975. Etude phytoécologique du pays baoulé méridional (Côte d'Ivoire centrale). Thèse Doct. Ingénieur, CNRS A.011957, Montpellier.

Jongkind CCH. 2004. Checklist of Upper guinnea forest species. In Biodiversity of West African forests, an Ecological Kouakou EN. 2007, Poorter L, Bongres F, Kouamé NF, Hawthorne WD (eds). Atlas of Woody plant Species, CABI Publishing: Cambridge; 447-447.

Koffi KJ, Kouassi AF, Yao CYA, Bakayoko A, Ipou IJ, Bogaert J. 2015. The 
present state of botanical investigations in Côte d'Ivoire. Biodiversity Informatics, 10(2): 56-64.

Kouamé FN. 1998. Influence de l'exploitation forestière sur la végétation et la flore de la forêt classée du Haut-Sassandra (Centre-Ouest de la Côte d'Ivoire). Thèse de $3 \mathrm{e}$ cycle, Université de Cocody, Abidjan.

Koulibaly AV. 2008. Caractéristiques de la végétation et dynamique de la régénération, sous l'influence de l'utilisation des terres, dans les mosaïques forêts-savanes des régions de Lamto et du Parc National de la Comoé en Côte d'Ivoire. Thèse de Doctorat, Université de CocodyAbidjan.

Lebrun JP. 1947. La Végétation de la Plaine Alluviale au Sud du Lac Edouard. Mission Lebrun J. 1937 - 1938. Inst. Parcs Nat. Congo Belge, Expl, Parc Nat: Congo Belge.

Lauginie F. 2007. Conservation de la nature et aires protégées en Côte d'Ivoire. CEDA/NEI.

Lykke AM, Sambou B. 1998. Structure, floristic composition, and vegetation forming factors of three vegetation types in Senegal. Nord. J. Bot., 18(2) : 129-140.

Lykke AM, Sambou B, 2002. Dynamique des espèces ligneuses dans une savane soumise aux feux dans le parc National du Delta du Saloum, Sénégal. Am. Bot. Afr. Ouest, 02: 67 - 78.

Lykke AM. 1998. Assessment of species composition change in savanna vegetation by means of woody plant size class distributions and local information. Biodiversity and Conservation, 7: $1261-1275$.

Mitja D. 1990. Influence de la culture itinérante sur la végétation d'une savane humide de Côte-d'Ivoire (BooroBorotou, Touba). Thèse de doctorat, Université de Paris.

Myers N, Mittermeier RA, Mittermeier CG, Da Fonseca GAB, Kent J. 2000.
Biodiversityhotspots for conservation priorities. Nature, 403 : 853-858.

Nusbaumer L, Gautier L, Chatelin C, Spichiger R. 2005. Structure et composition floristique de la Forêt Classée du Scio (Côte d'Ivoire). Etude descriptive et comparative. Candollea 60(2): 393-443.

Piélou EC. 1966. Species diversity and pattern diversity in the study of ecological succession. J. Theor. Biol., 10: 370383.

Planchon O. 1989. Le relief les paysage et les formes d'érosion linéaire, leur importance dans le fonctionnement d'un petit bassin versant (BooroBorotou, Côte d'Ivoire). Thèse de doctorat, USTL, Montpellier.

Sangne YC. 2009. Dynamique du couvert forestier d'une aire protégée soumise aux pressions anthropiques : cas de la Forêt Classée de Téné dans le Département d'Oumé (Centre-ouest de la Côte d'Ivoire). Thèse de Doctorat, Université de Cocody-Abidjan.

Shannon CE, Weaver W. 1949. The Mathematical Theory of Communications. Univ. Illinois Press: Urbana (USA).

Ter Braak C. 1985. Correspondence analysis of incidence and abundance data: properties in terms of unimodal response model. Biometrics, 41: 859873.

Teyssèdre A. 2004. Vers une sixième grande crise d'extinctions? Biodiversité et changements globaux: enjeux de société et défis pour la recherche. Ministère des Affaires EtrangèresADPF, Paris, 24-36.

Tra Bi FH. 1997. Utilisation des plantes par l'homme, dans le Haut-Sassandra et de Scio, en Côte d'Ivoire. Thèse de $3 \mathrm{e}$ cycle. Université de Cocody, U.F.R. Biosciences, Abidjan.

UICN. 2015. IUCN red list of threatened species. Version 2015.1. <www.iucnredlist.org>. Downloaded on 17 March 2016. 